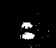
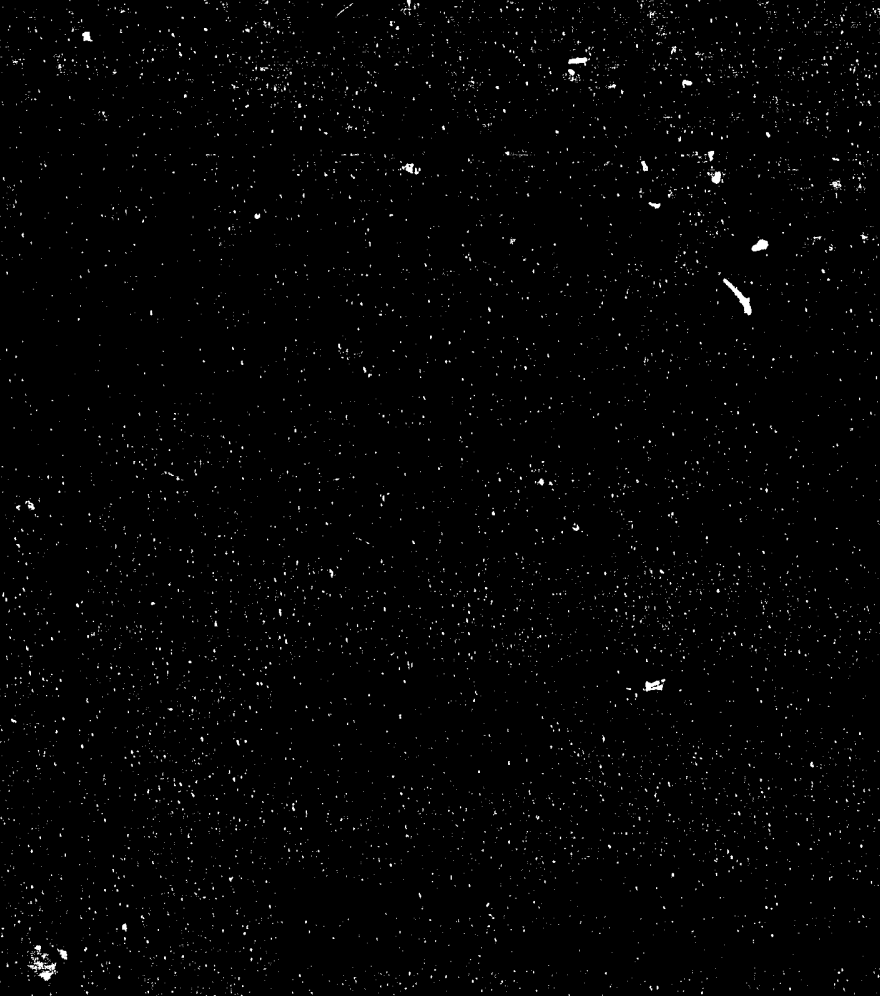

$\$$
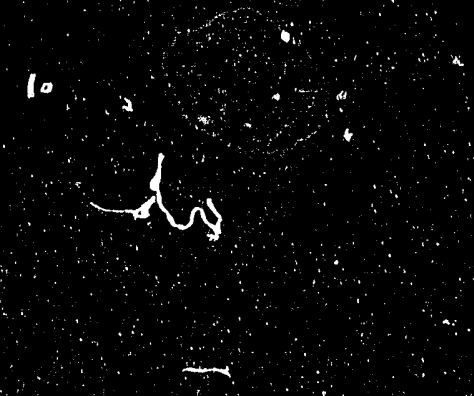

3

$\mathbf{t}$

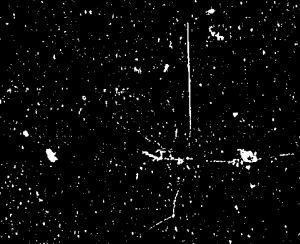




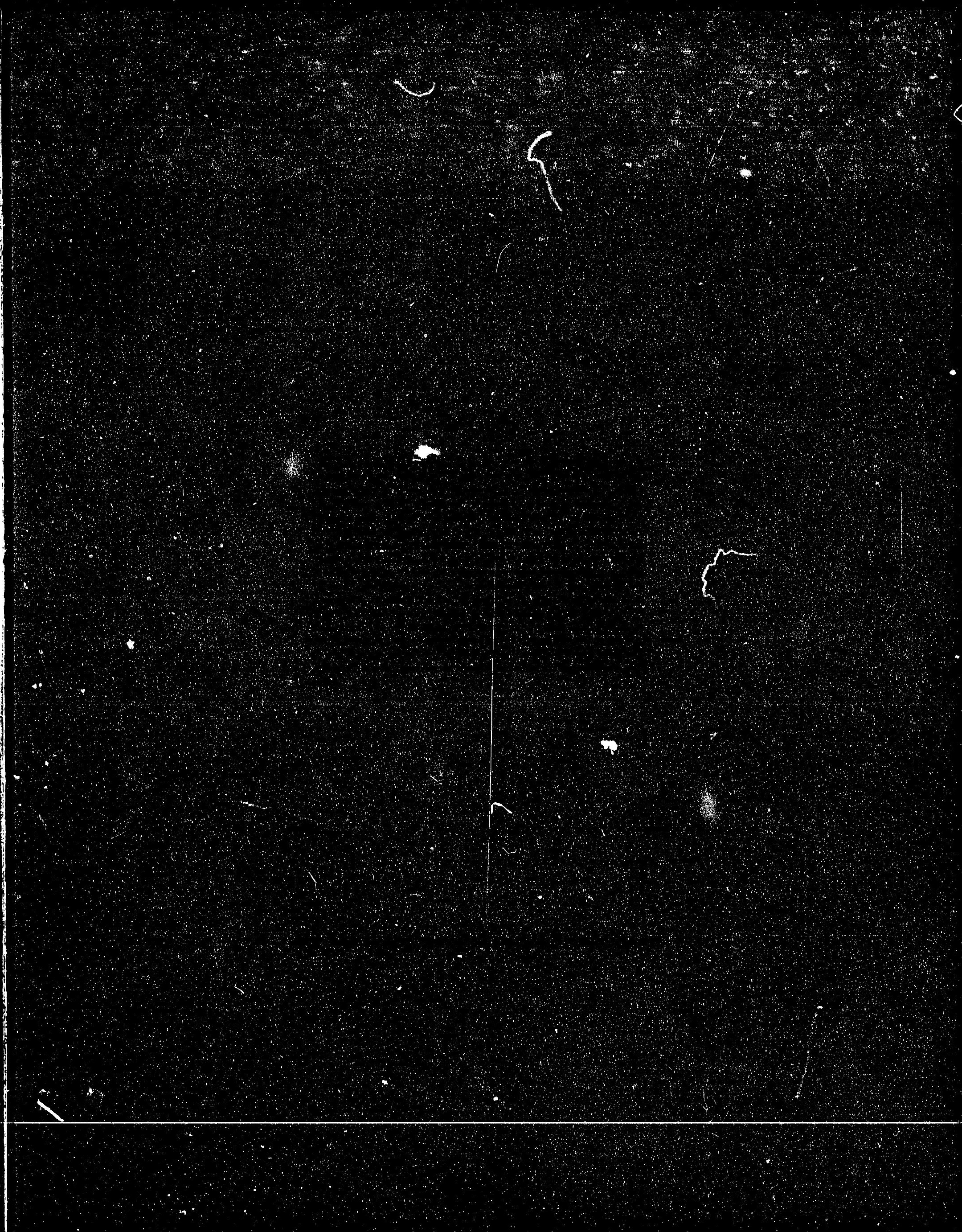


LBL-33227

UC-410

LSGN-110

\title{
DESIGN AND PRELIMINARY RESULTS FOR A FAST BIPOLAR RESONANT DISCHARGE PULSER USING SCR SWITCHES FOR DRIVING THE INJECTION PUMP MAGNETS AT THE ALS
}

\author{
GREG STOVER and LOU REGINATO \\ ACCELERATOR and FUSION RESEARCH DIVISION \\ Lawrence Berkeley Laboratory \\ University of California \\ Berkeley, CA 94720
}

MAY 1993

This work was supported by the Director, Office of Energy Research, Office of Basic Energy Sciences ${ }_{r}$ Materials Sciences Division, of the U.S. Department of Energy under Contract No. DE-AC03-76SF00098. 


\title{
Design and Prelıminary Results for a Fast Bipolar Resonant Discharge Pulser Using SCR Switches for Driving the Injection Bump Magnets at the ALS*
}

\author{
Greg Stover and Lou Reginato \\ Lawrence Berkeley Laboratory, University of California \\ 1 Cyclotron Road, Berkeley, CA 94720 USA
}

\section{Abstract}

A fast (4.0 us half period) resonant discharge pulser using SCRs has been designed and constructed to drive the injection bump magnet system at the Advanced Light Source (ALS). The pulser employs a series-parallel arrangement of Silicon Controlled Rectifiers (SCRs) that creates a bipolar high voltage $(+/-10 \mathrm{KV})$, high peak current (6600 amps.) and a high di/dt (6000 amp/us) switch network that discharges a capacitor bank into the magnet load. Fast recovery diodes in series with the SCRs significantly reduces the SCR turn-off time during the negative current cycle of the magnet. The SCR switch provides a very reliable and stable alternative to the gas filled Thyratron. A very low impedance transmission system allows the pulser system to reside completely outside the storage ring shielding wall.

\section{INTRODUCTION}

Injection into the storage ring (SR) employs a well proven technique of stacking in radial phase space. Prior to injection, the SR closed orbit is deflected ( $15 \mathrm{~mm}$ max.) in a sinusoidal manner towards the injection septum by two pairs of bump magnets. At the peak of the sinusoid wave form a packet ( 1 to 10 bunches) of $1.5 \mathrm{MeV}$ electrons is extracted from the booster synchrotron, transported through the extraction septa, the transfer line, the injection septa, and into the storage ring. This paper will discuss the design of the electrical system that drives the SR bump magnets.

\section{SYSTEM DESIGN}

\section{A. Magnet Specifications}

Each bump magnet is constructed in a window-frame geometry with a nickel-zinc ferrite core and is energized by a single-turn copper conductor. For ease of maintenance and cost all the magnets were designed to fit outside the vacuum chamber. The chamber is made of a high-alumina ceramic and has a Titanium Molybdenum semiconductive coating that has been thermally evaporated on the inner surface of the tube. The coating provides a consistent and predictable electrical impedance to the electron beam with enough resistive loss to minimize the magnetic field cancellation effects.

The purpose of the SR bump magnets driver is to produce the temporary (4 us) desired beam deflection to accept the electron packet while minimizing induced coherent betatron motion and simultaneously avoiding a fourth turn collision with the injection septum.

\footnotetext{
*This work was supported by the Director, Office of Energy Research, Office of Basic Energy Sciences, Materials Sciences Division of the U.S. Department of Energy, under Contract No. DEAC03-76SF00098.
}

\section{B. Electrical Specifications}

The physics of the injection process produced the following electrical driving parameters for each magnet; a half sinusoidal current wave form with a period of 4.0 us, a peak current of 3300 amperes, a reactive voltage of 6000 volts, and a maximum reverse recovery amplitude of $<1.0 \%$ of the peak forward current in the magnets.

\section{Overall System Layout}

The sinusoidal current wave form is generated by the resonant discharge of a capacitor bank through an electronic switch into the predominantly inductive magnet load. The hydrogen thyratron is the switching device usually specified to handle the voltage and current levels required for this system. Unfortunately, thyratrons have reliability problems and lifetimes that are limited to a few thousand hours or less[1]. In the face of this situation, we decided to develop a solid-state switch using off-the-shelf, very reliable, high voltage, high $\mathrm{di} / \mathrm{dt}$, Silicon Controlled Rectifiers (SCRs).

The basic topology for the pulse system is shown in Figure 1. The bump magnets are connected by a very low inductance transmission network to four parallel bipolar SCRcapacitor discharge modules that are shown in Figure 2. The modules are charged by two opposite polarity high voltage power supplies.

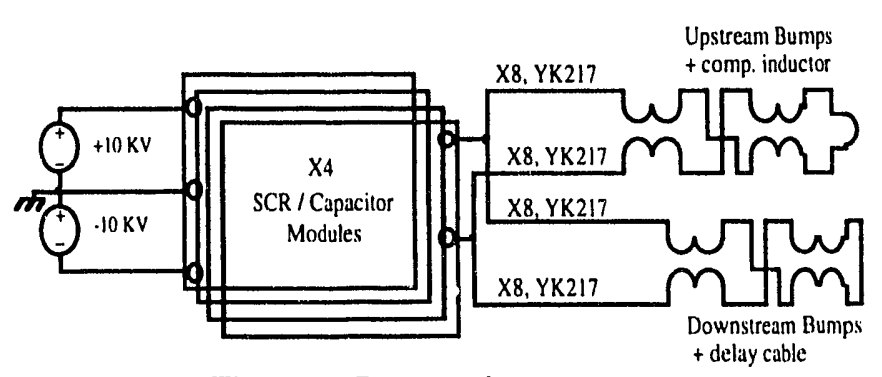

Figure 1. Bump pulser system.

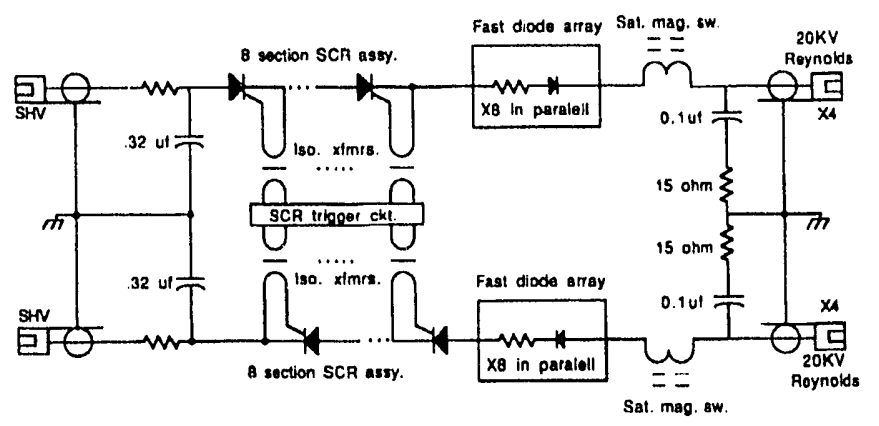

Figure 2. One of four bipolar SCR-capacitor discharge modules. 


\section{The Load Configuration}

Great care was taken to minimize any potential offsets in the beam closed orbit during the injection cycle. Precise magnetic field correspondence between the two magnet groups was accomplished through the exacting construction of each magnet and the series-parallel connection of the magnets to the discharge modules. Furthermore, the "downstream". magnets have an additional $5.3 \mathrm{ft}$. of cable to make up for the electron "time of flight" between the two magnet groups. A small balancing inductor was added to the "upstream" group to compensate for the inductance of the extra "downstream" cable.

\section{E. Low Inductance Transmission System}

For ease of maintenance and accessibility we chose to locate all the active components of the pulse system outside of the SR shielding wall. The average cable run length is approximately 60 feet from the magnet load to the driver. In and effort minimize the inductive voltage drop and the overall cost of all the high voltage charging components a very low inductance transmission system was constructed. As shown in Figure 1, each of the magnet terminals is connected to the discharge modules by narallel low inductance cables. A total of 32 cables are $\mathrm{cr}_{1}$, connected from the four magnet terminals to the four modules to further enhance the current distribution. The coax cable, YK217 [2], has linear inductance $33 \mathrm{NH} / \mathrm{ft}$ and cable impedance of approximately $15 \mathrm{ohms}$.

\section{F. The SCR-Capacitor Discharge Modules}

The series/parallel connection of the load sets the nominal peak load current at 6600 amperes. For a sinusoidal wave form this generates a maximum $\mathrm{di} / \mathrm{dt}$ value of $5.2 \mathrm{Kilo}$ amps./sec at the beginning of the discharge cycle. This $\mathrm{di} / \mathrm{dt}$ rating is much higher than any single commercial SCR can physically support. As shown in Figure 1 problem was solved by dividing the load current equally between four parallel pulsers.

The SCR used is a WESTCODE, R200CH18F2K0 [3] that has a $34 \mathrm{~mm}$ die diameter and employs a interdigitated amplified gate structure. The 1800 volt blocking voltage keeps the number of stacked devices to a manageable number of 8 and the di/dt spec. of $1500 \mathrm{amps}$./us limits the number of required parallel modules to four. Recent articles[4] and our operating results indicate that the vendor specified $\mathrm{di} / \mathrm{dt}$ value is quite conservative.

The long recovery time, $\mathrm{Tq}=40$ us, of the $\mathrm{R} 200$ is shortened to $300 \mathrm{~ns}$ by the insertion of a parallel array of high voltage fast recovery diodes in series with the SCR string. See Figure. 2. In reality, the system reverse recovery time is effectively forced to zero by the in-series addition of low inductance saturable magnetic reactors. Any remaining reverse recovery transients are snubbed by the shunt $\mathrm{RC}$ network connected to the outputs.

Moreover, these reactors improve the SCR turn-on and $\mathrm{di} / \mathrm{dt}$ capabilities. During the first $300 \mathrm{~ns}$ of the turn-on cycle the majority of the applied voltage is dropped across the as yet unsaturated reactors allowing time for extra charge injection into the gate structure of the SCRs. This condition avoids initial current crowding effects during turn-on that can destroy these devices. When the reactors saturate the SCRs are effectively $O N$ and ready to conduct current.

The SCR strings are mounted in a compression clamp holds all eight devices. The voltage across the stack is graded by a string of 20 megohm resistors. Each SCR has an RC snubber in shunt from anode to cathode. High frequency torroidal 3E2A ferrite transformers isolate the individual SCR gates from the low level trigger circuitry. The peak trigger current into the each gate of each SCR is approximately 20 amperes.

Monitoring of the discharge module current and voltage is accomplished through in-house designed Rogowski belts and high frequency dividers. The field of each bump magnet is measured by individual $B$ dot loops and passive integrators.

\section{OPERATIONAL RESULTS}

The photo in Figure 3 is the exact overlay of two oscilloscope wave forms of the magnet currents measured simultaneously in the "upstream" and "downstream" bump magnets. The current was measured with two separate Pearson current transformers matched to better that $0.1 \%$. The identical nature of the wave forms confirms the validity of the current sharing schemes employed by this system. The reverse recovery wave form is smooth but larger than the $1.0 \%$ specified. IsSPICE [5] circuit simulations indicate that the load snubber impedance can be reduced to minimize the amplitude of the recovery.

The upper trace in Figure 4 is the magnetic field measured by a 3 turn $B$ dot loop and a passive integrator. The discrepancy in shape between the magnet current and the field wave forms is do to the bandwidth limit of the field measuring electronics. The lower trace is the output current from one of the four SCR switch units. The current wave forms of the other three units are very closely matched to the form shown. The high frequincy ringing superimposed on the discharge pulse has a nominal period of $800 \mathrm{~ns}$. Due to the ringing peak $\mathrm{di} / \mathrm{dt}$ has been measured as high as $3300 \mathrm{amps}$./us or twice the specified maximum listed by the manufacturer. A SPICE analysis has confirmed the suspected parasitic components of this ringing to be a $C, L, C$ resonance excited by the initial turnon transient. The $l_{4}$ mped components are the distributed series inductance of the SCR switch interacting with the series inductance and shunt capacity of the transmission cable.

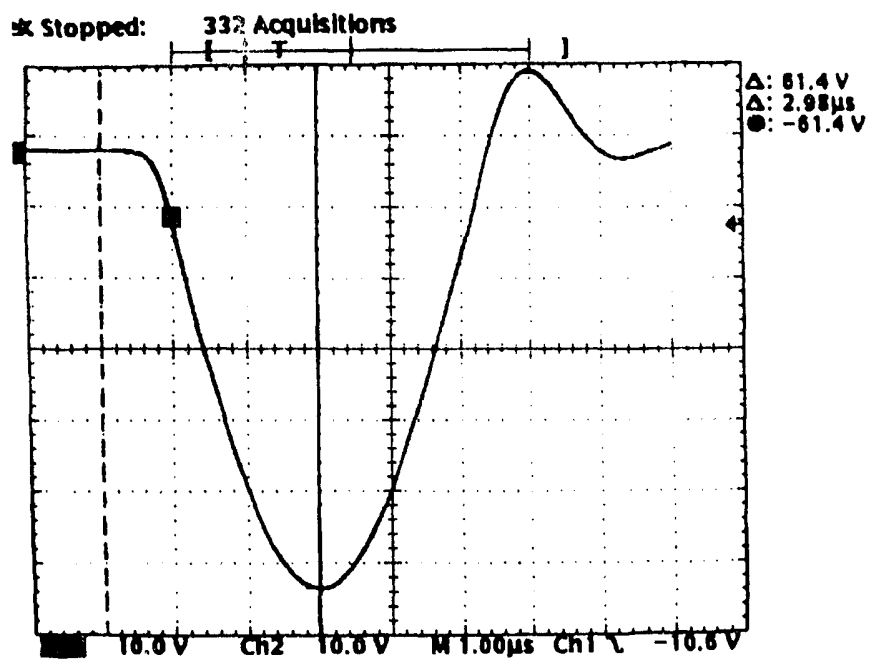

Figure 3. Magnet current wave forms (overlaid) in "upstream" and "downstream" bump magnets (100 amps. /div.). 


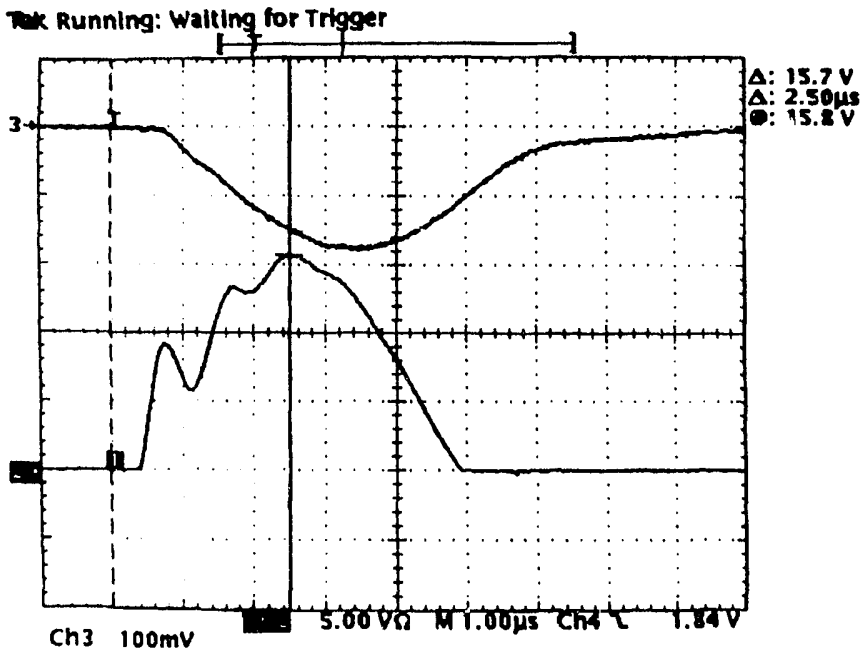

Figure 4. Magnetic field (upper trace, 666 gauss/div.) and pulser output current

(lower trace, 500 amps. /div.)

The SPICE model wave forms of the pulser output current (upper trace) and magnet load current (lower trace) are shown in Figure 5. The model has been empirically adjusted to give reasonably good agreement with the actual wave forms shown in Figures 3 and 4 . The noticeable discrepancy is in the amplitude and duration of the reverse recovery cycle. This difference is in part due to the absence of an equivalent saturable reactor in the SPICE model. The reactor will be included in future models.

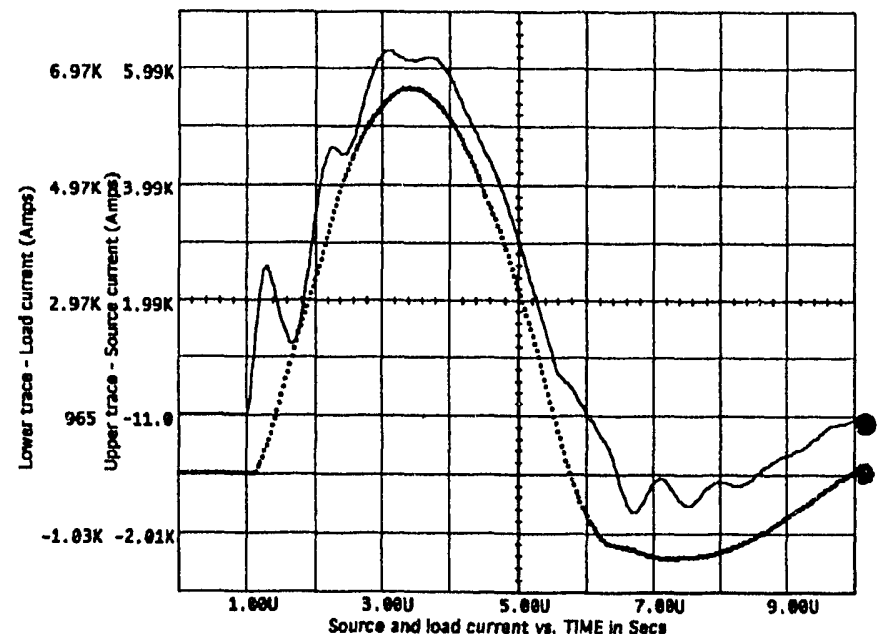

Figure. 5. SPICE model output wave forms: Pulser output current (upper trace, 1000 amps./div.) and magnet load current (lower trace, $1000 \mathrm{amps}$. /div.)

One solution to the resonance problem is shown in Figure 6. The ringing is almost eliminated and the reverse recovery wave form is greatly improved. In this case the number of transmission cables was halved from 32 to 16 and the snubber shunt capacitance was increased by a factor of 10 . At present we still considering at the most optimal solution.

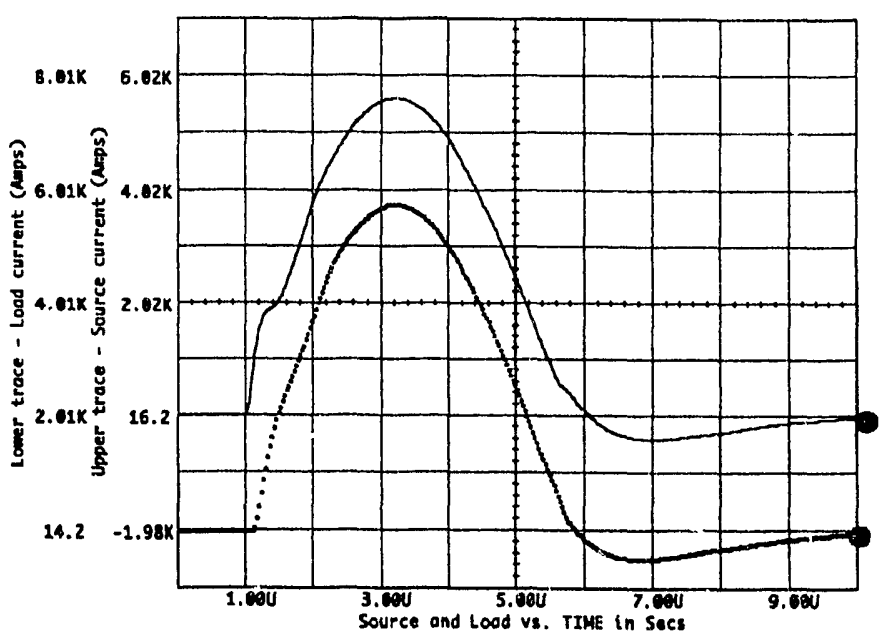

Figure 6. SPICE model output wave forms: Pulser output current (upper trace, 1000 amps./div.) and magnet load current

(lower trace, $1000 \mathrm{amps}$. /div.)

\section{CONCLUSION}

The bump pulser system has been operating at the peak current of 6600 amps since the initial commissioning phases of the ALS in January. Despite the excessive di/dt stresses placed on the SCR's, the system has operated without fault up to shutdown date of May l.

\section{REFERENCES}

[1] B.T. Merritt, F. R. Dreifuerst, "A New, High-Power, Solid State Switch Outperforms Thyratron Tubes" E\&TR Sept.Oct. 1991.

[2] Manufactured by Belden Wire and Cable (special order).

[3] Manufactured by WESTCODE Semiconductors Limited of England.

[4] J.L. Hudgins, V.A. Sandaran, W.M. Portnoy, and K.M. Marks "High di/dt Switching with Thyristors" Dept. of Electrical and Computer Engineering, University of South Carolina, Columbia, SC 29208.

[5] Trademark of Intusoft, P.O. Box 710 San Pedro, CA. 90733-0710 (310) 833-0710. 


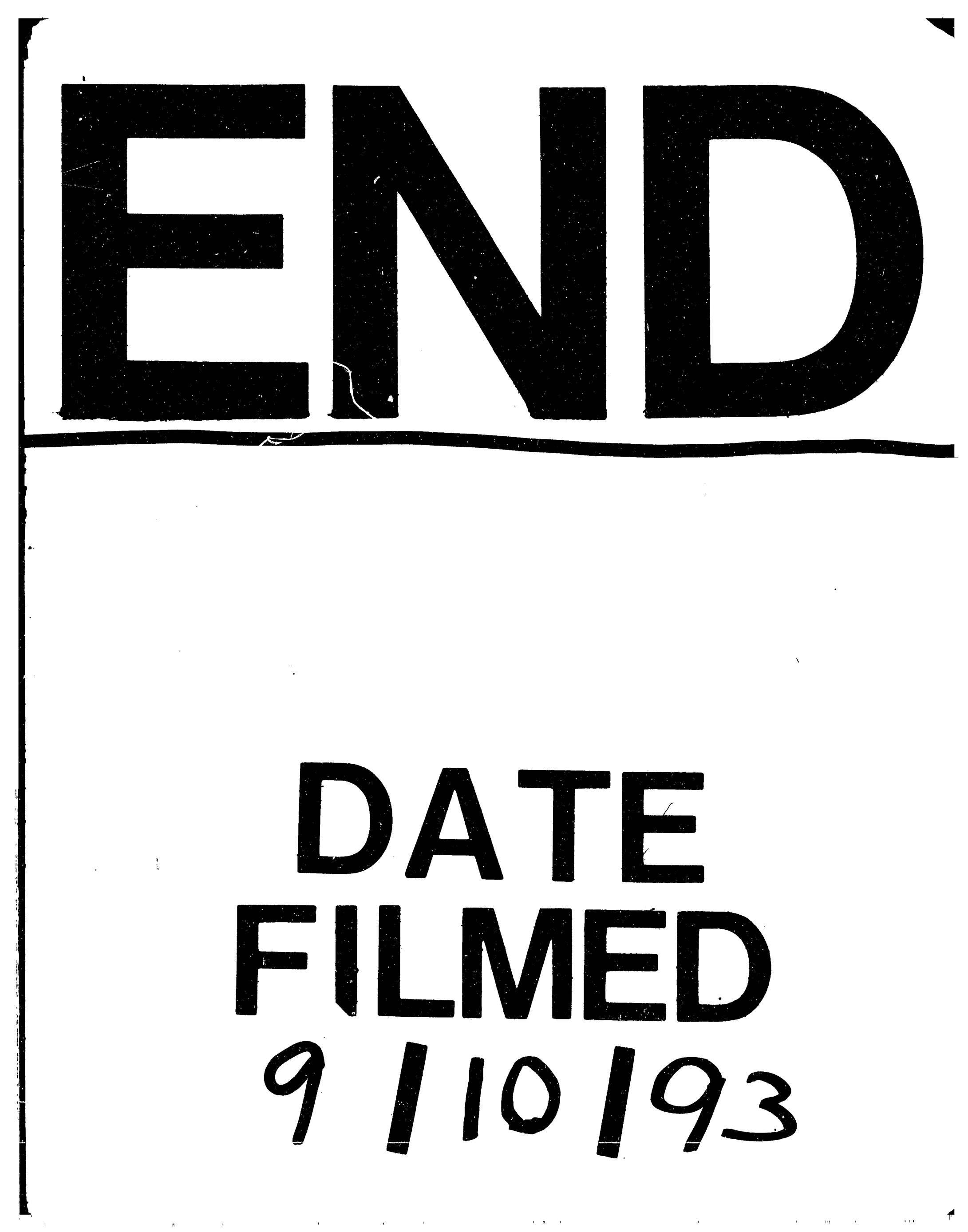


